1 Lateral Preoptic Area Neurons Signal Cocaine Self-Administration Behaviors

2 Kevin R. Coffey ${ }^{2}$, Vaishnavi Venkat ${ }^{1}$, Mark O. West ${ }^{1}$, David J. Barker ${ }^{1, *}$

$4 \quad{ }^{1}$ Department of Psychology, Rutgers, The State University of New Jersey.

$5 \quad{ }^{2}$ Department of Psychiatry and Behavioral Science, University of Washington School of

6 Medicine

*Correspondance : David J. Barker. Department of Psychology, Rutgers, the State University

9 of New Jersey. 152 Frelinghuysen Road, Piscataway, NJ 08854.

10 Email: David.Barker@rutgers.edu

12 Running title: Preoptic Drug-Seeking

13 Word Count- Main Text and Figure Captions-3415 words; Abstract-152 words.

\title{
21 Acknowledgements
}

22 This study was supported by the National Institute on Drug Abuse Grants DA043572 (DJB) and

23 DA06886 (MOW). The funders had no role in study design, data collection, data analysis,

24 decision to publish, or manuscript preparation. MW and DJB conceptualized the project. DJB

25 and KRC performed the surgeries, electrophysiological recordings, and the histology. DJB, and

26 VV Analyzed the data. KRC and DJB wrote the manuscript with contributions from all co-

27 authors. All authors have seen the manuscript and approved it for publication. The authors

28 declare that they do not have any conflicts of interest (financial or otherwise) related to the data

29 presented in this manuscript. 


\section{Abstract}

The lateral preoptic area is implicated in numerous aspects of substance use disorder. In

34 particular, the lateral preoptic area is highly sensitive to the pharmacological properties of

35 psychomotor stimulants, and its activity promotes drug-seeking in the face of punishment and

36 reinstatement during abstinence. Despite the lateral preoptic areas complicity in substance use

37 disorder, how precisely lateral preoptic area neurons encode the individual components of drug

38 self-administration has not been ascertained. To bridge this gap, we examined how the firing of

39 single lateral preoptic area neurons correlates with three discrete elements of cocaine self-

40 administration: 1) drug-seeking (pre-response), 2) drug-taking (response), and 3) receipt of the

41 cocaine infusion. A significant subset of lateral preoptic area neurons responded to each

42 component with a mix of increases and decreases in firing-rate. A majority of these neurons

43 encoded the operant response with increases in spiking, though responses during the drug-

44 seeking, taking, and reciept windows were highly correlated.

46 Keywords: Hypothalamus, Preoptic, Cocaine, Addiction, Electrophysiology 
Introduction

The lateral preoptic area (LPO) is a hypothalamic structure comprised of glutamate and GABA neurons(Barker et al., 2017; Geisler et al., 2007; Jones, 2005) with diverse and wideranging connections to limbic and limbic-related structures such as the lateral habenula ( $\mathrm{LHb}$ ) (Barker et al., 2017; Kowski et al., 2008; Mok \& Mogenson, 1972; Yetnikoff et al., 2015; Zahm \& Root, 2017), the dorsal raphe (DRN) (Peyron et al., 1997; Weissbourd et al., 2014), ventral tegmental area (VTA) (Geisler et al., 2007; Geisler \& Zahm, 2006; Yetnikoff et al., 2015), rostromedial tegmental nucleus (RMTg) (Jhou et al., 2009; Yetnikoff et al., 2015), and Locus Coeruleus (LC) (Luppi et al., 1995).

The LPO participates in a wide variety of behaviors from general locomotion(Reichard et al., 2019; Shreve et al., 1989; Shreve \& Uretsky, 1991; Subramanian et al., 2018; Zahm et al., 2014) and thirst(Saad et al., 1996), to reward-seeking(Gordon-Fennell et al., 2020) and aversive processing(Barker et al., 2017; Briski \& Gillen, 2001). Most notably, the LPO supports strong intracranial self-stimulation(Bushnik et al., 2000; Gallistel et al., 1985; Nakahara et al., 1999) and excitotoxic lesions of the LPO attenuate the rewarding efficacy of medial forebrain bundle self-stimulation(Arvanitogiannis et al., 1996).

A growing body of evidence suggest that the LPO plays a central role in substance use disorder. The LPO and neighboring portions of the substantia innominata participate in the locomotor activation that occurs following the administration of psychomotor stimulants(Swerdlow et al., 1986), and acute amphetamine administration elicits increased c-fos expression in the LPO(Colussi-Mas et al., 2007), especially in LPO efferents to the VTA. Our previous work has shown that LPO neurons are highly sensitive to the pharmacological properties of cocaine, with some neurons tracking ongoing changes in bodily levels of cocaine(Barker et al., 2015).

Perseverative motivated behavior despite conflict is a key trait of substance use

72 disorders(Ettenberg, 2004) that promotes drug relapse(Cooper et al., 2007). Recent evidence has

73 underscored the LPOs role in driving motivated behaviors in the face of conflict(Reichard et al.,

74 2019). Pharmacological stimulation or inhibition of the LPO disrupts the effects of punishment

75 on future cocaine-seeking behavior and stimulating the LPO is capable of driving cocaine 
76 reinstatement, likely through excitation of VTA dopamine neurons and inhibition of VTA

77 GABA neurons(Gordon-Fennell et al., 2020).

Here, we seek to determine precisely which components of cocaine self-administration are processed by the LPO, and how those components are encoded. We predict that if LPO

80 neurons participate in cocaine self-administration, they will exhibit firing rate modulations that

81 correlate with particular components of the behavior. To test this hypothesis, we examined how

82 the firing of single LPO neurons correlates with drug-seeking (pre-response), drug-taking

83 (response), and receipt of the cocaine infusion(Coffey et al., 2015; Root et al., 2013). A

84 significant subset of LPO neurons responded to each component with a mix of increases and

85 decreases in spiking. A majority of these neurons encoded the operant response with increased

86 firing, though responses during the drug-seeking, taking, and reciept windows were highly

87 correlated.

Materials and Methods

89 All protocols were performed in compliance with the Guide for the Care and Use of Laboratory

90 Animals (NIH, Publication 865-23) and have been approved by the Institutional Animal Care

91 and Use Committee, Rutgers University.

\section{Subjects and Surgery}

93 Male Long-Evans rats ( $\mathrm{n}=18$, Charles River, Raleigh, NC) were individually housed.

94 All animals were provided ad libitum access to water and sufficient food to bring them to a pre-

95 operative weight of $\sim 330 \mathrm{~g}$. Subjects were anesthetized with sodium pentobarbital (50 mg/kg,

96 i.p.; Abbott Laboratories, North Chicago, IL, USA) and administered atropine methyl nitrate (10

$97 \mathrm{mg} / \mathrm{kg}$, i.p.; Sigma, St. Louis, MO, USA) and penicillin G (75,000 U/0.25 ml, IM). Anesthesia

98 was maintained with sodium pentobarbital (5-10 mg/kg, IP) and ketamine hydrochloride (60

$99 \mathrm{mg} / \mathrm{kg}$, IP; Fort Dodge Laboratories, Fort Dodge, IA, USA). A catheter was implanted into the

100 right jugular vein and exited through a J-shaped stainless steel cannula. An array of either 16

101 microwires (Microprobes, MD, USA) arranged in two parallel rows of 8 wires with the medial

102 eight wires targeting the LPO or 2 bilateral arrays each with 2 parallel rows of four wires all

103 targeting the LPO were implanted. Both the J-shaped cannula and array headstage were secured

104 to the skull using 5 jewelers' screws and dental cement. Animals were housed in the self-

105 administration chamber for the remainder of the experiment and were allowed to recover for at 
least 7 days prior to training. Chambers were located inside a soundproof, ventilated box that

107 was supplied with white noise $(75 \mathrm{~dB})$. During all hours other than self-administration sessions, a

$108200 \mu \mathrm{L}$ infusion of heparinized-saline was delivered every $25 \mathrm{~min}$ by a computer controlled

109 syringe pump, through a leash connected to the animal's headstage 24/7, to preserve catheter

110 patency. Occasionally, a brief period of anesthesia was induced by an infusion of methohexital

111 sodium $(10 \mathrm{mg} / \mathrm{kg}, \mathrm{IV})$ in order to check the animal's patency.

\section{Self-administration}

113 Daily cocaine self-administration sessions began with insertion of a nonretractable glass

114 lever on the side wall of the Plexiglas chamber and illumination of the house light within the

115 sound attenuating chamber. To initiate the first trial, a stimulus light over the lever was

116 illuminated. One lever press in the presence of the stimulus light immediately extinguished the

117 stimulus light and initiated an intravenous infusion of cocaine $(0.24 \mathrm{mg} / 0.2 \mathrm{ml}$ infusion over 7.5

$118 \mathrm{~s})$ and a $7.5 \mathrm{~s}$ tone $(3.5 \mathrm{kHz}, 70 \mathrm{~dB})$ which co-terminated with the infusion pump. The stimulus

119 light remained off for $40 \mathrm{~s}$ during which lever presses had no programmed consequences.

120 Sessions lasted until 50 infusions had been earned or $6 \mathrm{~h}$ had elapsed, whichever occurred first.

All rats were trained for at least 2 weeks prior to electrophysiological recordings (Figure

123 1A). Neural signals were led from the rat's head, through a harness, and then to a fluid and

124 electronic swivel (Airflyte Electronics, Bayonne NJ; Plastics One Inc., Roanoke VA). Electrical

125 signals were sampled (50 kHz/wire), digitized, time stamped ( $0.1 \mathrm{~ms}$ resolution), and recorded

126 using SciWorks software (DataWave Technologies, Longmont CO). Self-administration during

127 recording sessions lasted until rats had earned 50 infusions, at which point contingencies ended

128 and the lever was removed. Neural recording sessions began 30 min prior to the start of self-

129 administration and continued for 1 additional hour after the termination of self-administration

130 contingencies (by removal of the glass lever). Each rat received 1-3 neural recording sessions in

131 order to record each of the 15 non-differential probes one time (5 or 15 probes recorded/session,

132 depending on hardware). 
Rats were euthanized with sodium pentobarbital (300 mg/kg, i.p.), and anodal current (50

$137 \mu \mathrm{A}$ for $4 \mathrm{~s}$ ) was passed through each electrode. Rats were then perfused with formalin-saline (n

$138=12)$ or $4 \%$ paraformaldehyde $(n=6)$. Brains were post-fixed overnight and then transferred to

139 a $30 \%$ sucrose solution. Sections were taken coronally $(50 \mu \mathrm{m})$ through the LPO. Tissue was

140 stained in a $5 \%$ potassium ferrocyanide and $10 \% \mathrm{HCl}$ and counterstained for antibodies against

141 calbindin D28k immunohistochemistry (ImmunoStar, Inc., Hudson WI), as described

142 previously(Coffey et al., 2015). Wire tracks for every wire in each array were traced from the

143 point at which they penetrated cortex and down to the lesion produced at the microwire tip. In

144 instances where the three-dimensional location of each and every microwire in the array was not

145 able to be accurately tracked, the rat's data were removed from the dataset. Experimenters blind

146 to results established the location of tracked microwire tips by overlaying microscope images

147 onto a stereotaxic atlas(Paxinos \& Watson, 2006) using Photoshop (Adobe, San Jose CA). Wires

148 were included for analysis if they were localized to the LPO. Any microwires localized within

$149100 \mu \mathrm{m}$ of any non-LPO brain region were excluded from analysis.

Isolation/discrimination of individual waveforms

Analysis of neural data was conducted using SciWorks software (DataWave

152 Technologies, Longmont CO). The software was used to isolate neural waveforms from ambient

153 noise and to discriminate different neurons recorded by the same microelectrode. Several

154 measures were taken to identify individual neurons on probes containing more than one

155 waveform. Isolation of neurons was based on multiple measures including spike height, valley

156 and peak amplitudes, valley and peak times, and voltage ranges at selected time points on the

157 ascending and descending limbs of the waveforms. Waveforms were included for analysis if (1)

158 waveforms presented with canonical patterns of neural activity including a clearly defined

159 waveform for the action potential, (2) the amplitude of putative neurons exhibited at least a 2:1

160 signal/noise ratio, (3) waveform parameters remained stable throughout the entire session, and

161 (4) an interspike interval (ISI) histogram showed that no action potential discharges occurred

162 during the neuron's natural refractory period (i.e., $\sim 1.4$ to $2 \mathrm{~ms}$ ). 
Change scores were calculated for three major event windows: 1) drug-seeking (predrug-taking (response) firing patterns, defined as the $500 \mathrm{~ms}$ period following a lever press, and 3) drug receipt (infusion-related) firing patterns, defined as the $500 \mathrm{~ms}$ period immediately following the end of the $7.5 \mathrm{~s}$ cocaine infusion. Baseline firing was defined as the time window

171 from $-10 \mathrm{~s}$ to -5 seconds relative to the lever press. Change scores were calculated as:

$$
\frac{(\text { Event } F R-\text { Baseline } F R)}{(\text { Event FR }+ \text { Baseline FR) }}
$$

For these event windows, we often observed a bi-directional response of LPO neurons, with a mixture of increases and decreases as compared to each neuron's baseline firing rate.

175 Thus, we operationally defined neurons exhibiting an 'increase' as those showing a two-fold or 176 greater change from baseline firing rates (change score $>0.33$ ) and 'decreasing' neurons as those

177 exhibiting a two-fold or greater decrease from baseline firing rates (change score $<-0.33$ ). In

178 accounting for the bi-directional nature of neuronal responses, the absolute values of change 179 scores were used for statistical comparisons. Examinations of these neuronal samples were 180 conducted with a one-sample t-test, comparing the population to a value of 'zero', which 181 corresponds to a change score representing no change from baseline.

182 Results

183 Out of the 288 total microwires implanted, a total of 42 recorded neurons were localized 184 in the lateral preoptic area (Figure 1B). The average on-drug firing rate of all LPO neurons 185 during baseline was $0.74 \pm 0.17$ impulses/ second, with the fastest firing neuron reaching 5.49 186 impulses/second.

\section{A Subset of LPO Neurons Encode Cocaine-Seeking}

The pre-press window encompasses preparatory, drug-seeking behaviors that are

189 proximal to the lever press, including approaches to the lever(Root et al., 2013). A small, but 190 significant number of neurons responded during the pre-press period $(\mathrm{t}(41)=5.74, \mathrm{p}<0.001$;

191 Figure 1 C). The responses of these neurons were heterogeneous, with LPO neurons exhibiting 192 either increases or decreases when compared to their baseline firing rates. In total, $11.90 \%$ of 
193 LPO neurons exhibited a pre-press increase $(n=5 / 42), 16.67 \%$ of neurons exhibited a decrease

$194(n=7 / 42)$ and $71.43 \%(n=30 / 42)$ exhibited no change when compared to baseline.

The drug taking period encompasses the moment that rats lever-press for cocaine, along with the presentation of a CS+ cue, and the start of the cocaine infusion. We observed that LPO neurons - as a population — responded at the time of the lever press $(\mathrm{t}(41)=7.52, \mathrm{p}<0.001$;

199 Figure 1D). LPO responses at the time of the lever press were again a mixture of increases or 200 decreases in firing rate when compared to baseline firing rates. More LPO neurons exhibited 201 increases at the time of the lever press $(21.43 \% ; n=9 / 42)$ as compared to the pre-press drug202 seeking period, but a similar number of neurons exhibiting decreases were observed (19.05\%; $203 \mathrm{n}=8 / 42)$. The remaining 59.53\% $(\mathrm{n}=25 / 42)$ showed little or no change in their response compared 204 to baseline.

The drug receipt period encompasses behavioral or neural responses that occur in response to the receipt of the full infusion. The population of LPO neurons was responsive to the receipt of the infusion $(\mathrm{t}(41)=6.07, \mathrm{p}<0.001$; Figure $1 \mathrm{E})$. The primary response following the end of the infusion was an inhibition of LPO neurons, with $16.67 \%(n=7 / 42)$ of LPO neurons

210 exhibiting decreases and only $4.76 \%(n=2 / 42)$ of neurons showing a post-infusion increase. Most 211 neurons exhibited little or no change in their firing rate compared to baseline $(78.57 \% ; n=33 / 42)$.

213 cocaine over longer time scales(Barker et al., 2015), but also suggest that rapid firing patterns

214 during self-administration behavior can be decoupled from long-lasting changes in neuronal

215 firing associated with the pharmacological effects of cocaine.

216 LPO Neuron Activity is Correlated Across Each Component of Cocaine Self-Administration

In examining peri-event responses for LPO neurons across the drug-seeking, drug taking,

218 and drug receipt event windows, we recognized that many neurons exhibited common responses

219 across multiple events. A graphical analysis of neurons inhibited at the time of the lever press 220 revealed that this subset of neurons exhibited a lengthy change in firing rate, in that they were 221 also inhibited at the time of the pre-press and infusion event windows. (Figure 2A). On the other 
222 hand, neurons that were excited at the time of the lever press also exhibited excitatory responses

223 during the pre-press window (Figure 2B) but exhibited a more transient change in firing rate and

224 appeared to be unresponsive or mildly inhibited at the end of the infusion.

Accordingly, we observed that responses across events were highly correlated. Responses

226 during the pre-press window were correlated with those at the lever press $\left(\mathrm{R}_{2}=0.16, \mathrm{P}<0.01\right.$;

227 Figure 2C), although the range of change scores during the pre-press window was far smaller.

228 Similarly, responses at the time of the pre-press were highly correlated with those at the end of

229 the infusion $\left(\mathrm{R}^{2}=0.17, \mathrm{p}<0.01\right.$;). As observed in the average response profile (Figure $\left.2 \mathrm{~A}\right)$, this

230 correlation appeared to be driven by long-lasting inhibitory responses. Finally, responses at the

231 lever press were correlated with those at the end of the infusion $\left(R^{2}=0.17, p<0.01\right.$; Figure $\left.2 E\right)$.

232 Similar to the relationship observed between the pre-press and lever press, the dynamic range for

233 change scores at the time of the infusion was much smaller.

234 Based on these findings, we conducted a graphical analysis of neurons that exhibited

235 operationally defined increases, decreases, or a mixture of responses across one or more events

236 ( $n=23$ of 42 neurons). This analysis suggested that most large neuronal changes were tied to the

237 time of the lever responses, with 8 out of the 23 neurons (34.8\% of responsive neurons)

238 responding only to the lever press, and an additional 9 neurons (39.1\%) also responding during

239 the pre-press window $(n=2 ; 8.7 \%)$, infusion window $(n=2 ; 8.7 \%)$, or both $(n=5 ; 21.7 \%)$. A small

240 subset of neurons responded only during the pre-press window $(n=3 ; 13 \%)$ or during both the

241 pre-press and infusion windows $(n=2 ; 8.7 \%)$, and only 1 neuron responded only at the time of

242 the infusion window (4.3\%). Overall, these results are consistent with the hypothesis that the

243 responses of LPO neurons are intimately tied with drug-taking responses.

\section{Discussion}

The LPO has been repeatedly linked to substance use disorder. Nonetheless, it has never

246 been explicitly demonstrated whether the firing of LPO neurons correlates with drug self-

247 administration (SA) behavior, and to which components of SA these neurons are responsive.

248 Here we provide evidence that subsets of LPO neurons are responsive across multiple events

249 related to operant SA, with most neurons directly encoding drug-taking (lever press) responses.

250 Further, modulation of a small subset of LPO neurons preceded the lever-press, indicating they

251 encode drug-seeking. The responses of LPO neurons were heterogeneous in nature, with neurons 
that were either excited or inhibited during the pre-press, lever press, and infusion windows.

253 Moreover, we observed that the responses of these neurons are complex, with several neurons

254 exhibiting long-lasting responses that begin prior to the lever press and continue through the end 255 of the infusion.

256 The presence of two opposing classes of LPO neurons is particularly interesting and may 257 relate to the heterogeneity in LPO neuronal populations. The LPO comprises both glutamatergic 258 and GABAergic neurons(Barker et al., 2017; Geisler et al., 2007; Jones, 2005), but lacks the 259 cholinergic neurons found in adjacent forebrain regions(Gritti et al., 1998). We recently observed 260 that LPO glutamate and GABA neurons play opposing roles in driving motivated behavior, at 261 least in their projections to the lateral habenula, with the activation of GABAergic neurons 262 producing reward and the activation of glutamatergic neurons driving aversion(Barker et al., 263 2017). Moreover, a recent study observed similar kinds of opposing responses in LPO 264 projections to the ventral tegmental area(Gordon-Fennell et al., 2019). The opposing neuronal 265 subtypes we observed during self-administration may represent a coordinated but opposing 266 response by LPO glutamate and GABA neurons.

267 Several prior studies have linked the LPO to locomotor responses or drug-induced 268 locomotor responses (Jones \& Mogenson, 1980; Mogenson \& Nielsen, 1983; Mogenson et al., 269 1983; Shreve \& Uretsky, 1989, 1991). These responses appear to depend on LPO local 270 glutamatergic or GABAergic signaling, with GABAergic and glutamatergic signaling playing 271 opposing roles(Shreve \& Uretsky, 1991). Based on these studies, one might conclude that the 272 long-lasting responses observed across the pre-press, lever press, and infusion events could relate 273 to locomotor activity during the peri-response window of self-administration sessions. However, 274 our prior work found no relationship between LPO firing rates and locomotor activity(Barker et 275 al., 2015). Moreover, one recent study failed to find an increase in general locomotor activity in a 276 context associated with operant responding(Gordon-Fennell et al., 2020), and additional work

277 has suggested that this locomotor activity may reflect fixed action patterns(Reichard et al., 2019).

278 When considered alongside the present data, these results suggest that the LPO plays a more 279 explicit role in goal-directed behaviors than was originally thought.

\section{Conclusion}

281 We provide here the first evidence that LPO neurons participate in multiple aspects of 282 drug self-administration, but most notably drug-taking behavior. Our findings also indicate that 
11

283 only a subset of LPO neurons participate in signaling during cocaine self-administration. It

284 would therefore be of great interest for future studies to examine whether these responsive

285 ensembles comprise a specific cell-type or project to a particular structure; projections to the

286 LHb(Barker et al., 2017), VTA(Gordon-Fennell et al., 2020; Gordon-Fennell et al., 2019), or

287 RMTG(Yetnikoff et al., 2015) are all compelling targets. Additionally, with the recent finding

288 that the LPO may play an important role in drug relapse and decision making during

289 relapse(Gordon-Fennell et al., 2020), more work is needed to determine the roles of specific LPO

290 neuronal subtypes in supporting drug-seeking behavior. A better understanding of these

291 processes could aid in the development of therapies to sustain drug abstinence in the face of

292 repeated conflict. 


\section{References}

Arvanitogiannis, A., Waraczynski, M., \& Shizgal, P. (1996). Effects of excitotoxic lesions of the basal forebrain on MFB self-stimulation. Physiol Behav, 59(4-5), 795-806. doi:10.1016/0031-9384(95)02157-4

Barker, D. J., Miranda-Barrientos, J., Zhang, S., Root, D. H., Wang, H.-L., Liu, B., . . Morales, M. J. C. r. (2017). Lateral preoptic control of the lateral habenula through convergent glutamate and GABA transmission. 21(7), 1757-1769.

Barker, D. J., Striano, B. M., Coffey, K. C., Root, D. H., Pawlak, A. P., Kim, O. A., . . . Function. (2015). Sensitivity to self-administered cocaine within the lateral preoptic-rostral lateral hypothalamic continuum. Brain Structure and Function, 220(3), 1841-1854.

Briski, K., \& Gillen, E. J. B. r. b. (2001). Differential distribution of Fos expression within the male rat preoptic area and hypothalamus in response to physical vs. psychological stress. 55(3), 401-408.

Bushnik, T., Bielajew, C., \& Konkle, A. T. (2000). The substrate for brain-stimulation reward in the lateral preoptic area. I. Anatomical mapping of its boundaries. Brain Res, 881(2), 103111. doi:10.1016/s0006-8993(00)02564-6

Coffey, K. R., Barker, D. J., Gayliard, N., Kulik, J. M., Pawlak, A. P., Stamos, J. P., \& West, M. O. (2015). Electrophysiological evidence of alterations to the nucleus accumbens and dorsolateral striatum during chronic cocaine self-administration. Eur J Neurosci, 41(12), 1538-1552. doi:10.1111/ejn.12904

Colussi-Mas, J., Geisler, S., Zimmer, L., Zahm, D. S., \& Berod, A. (2007). Activation of afferents to the ventral tegmental area in response to acute amphetamine: a double-labelling study. Eur J Neurosci, 26(4), 1011-1025. doi:10.1111/j.1460-9568.2007.05738.x

Cooper, A., Barnea-Ygael, N., Levy, D., Shaham, Y., \& Zangen, A. (2007). A conflict rat model of cue-induced relapse to cocaine seeking. Psychopharmacology (Berl), 194(1), 117-125. doi:10.1007/s00213-007-0827-7

Ettenberg, A. (2004). Opponent process properties of self-administered cocaine. Neurosci Biobehav Rev, 27(8), 721-728. doi:10.1016/j.neubiorev.2003.11.009

Gallistel, C. R., Gomita, Y., Yadin, E., \& Campbell, K. A. (1985). Forebrain origins and terminations of the medial forebrain bundle metabolically activated by rewarding stimulation or by reward-blocking doses of pimozide. J Neurosci, 5(5), 1246-1261.

Geisler, S., Derst, C., Veh, R. W., \& Zahm, D. S. (2007). Glutamatergic afferents of the ventral tegmental area in the rat. $J$ Neurosci, 27(21), 5730-5743. doi:10.1523/JNEUROSCI.001207.2007 
Geisler, S., \& Zahm, D. S. (2006). Neurotensin afferents of the ventral tegmental area in the rat:[1] re-examination of their origins and [2] responses to acute psychostimulant and antipsychotic drug administration. European Journal of Neuroscience, 24(1), 116-134.

Gordon-Fennell, A., Gordon-Fennell, L., Desaivre, S., \& Marinelli, M. (2020). The Lateral Preoptic Area and Its Projection to the VTA Regulate VTA Activity and Drive Complex Reward Behaviors. Front Syst Neurosci, 14, 581830. doi:10.3389/fnsys.2020.581830

Gordon-Fennell, A. G., Will, R. G., Ramachandra, V., Gordon-Fennell, L., Dominguez, J. M., Zahm, D. S., \& Marinelli, M. (2019). The Lateral Preoptic Area: A Novel Regulator of Reward Seeking and Neuronal Activity in the Ventral Tegmental Area. Front Neurosci, 13, 1433. doi:10.3389/fnins.2019.01433

Gritti, I., Mariotti, M., \& Mancia, M. (1998). GABAergic and cholinergic basal forebrain and preoptic-anterior hypothalamic projections to the mediodorsal nucleus of the thalamus in the cat. Neuroscience, 85(1), 149-178. doi:10.1016/s0306-4522(97)00573-3

Jhou, T. C., Geisler, S., Marinelli, M., Degarmo, B. A., \& Zahm, D. S. J. J. o. C. N. (2009). The mesopontine rostromedial tegmental nucleus: a structure targeted by the lateral habenula that projects to the ventral tegmental area of Tsai and substantia nigra compacta. 513(6), 566-596.

Jones, B. E. (2005). From waking to sleeping: neuronal and chemical substrates. Trends Pharmacol Sci, 26(11), 578-586. doi:10.1016/j.tips.2005.09.009

Kowski, A. B., Geisler, S., Krauss, M., \& Veh, R. W. (2008). Differential projections from subfields in the lateral preoptic area to the lateral habenular complex of the rat. J Comp Neurol, 507(4), 1465-1478. doi:10.1002/cne.21610

Luppi, P. H., Aston-Jones, G., Akaoka, H., Chouvet, G., \& Jouvet, M. (1995). Afferent projections to the rat locus coeruleus demonstrated by retrograde and anterograde tracing with cholera-toxin B subunit and Phaseolus vulgaris leucoagglutinin. Neuroscience, 65(1), 119-160. doi:10.1016/0306-4522(94)00481-j

Mok, A. C., \& Mogenson, G. J. (1972). An evoked potential study of the projections to the lateral habenular nucleus from the septum and the lateral preoptic area in the rat. Brain Res, 43(2), 343-360. doi:10.1016/0006-8993(72)90392-7

Nakahara, D., Ishida, Y., Nakamura, M., Kuwahara, I., Todaka, K., \& Nishimori, T. (1999). Regional differences in desensitization of c-Fos expression following repeated selfstimulation of the medial forebrain bundle in the rat. Neuroscience, 90(3), 1013-1020. doi:10.1016/s0306-4522(98)00510-7

Paxinos, G., \& Watson, C. (2006). The rat brain in stereotaxic coordinates: hard cover edition: Elsevier.

Peyron, C., Petit, J.-M., Rampon, C., Jouvet, M., \& Luppi, P.-H. J. N. (1997). Forebrain afferents to the rat dorsal raphe nucleus demonstrated by retrograde and anterograde tracing methods. $82(2), 443-468$. 
Reichard, R. A., Parsley, K. P., Subramanian, S., Stevenson, H. S., Schwartz, Z. M., Sura, T., \& Zahm, D. S. (2019). The lateral preoptic area and ventral pallidum embolden behavior. Brain Struct Funct, 224(3), 1245-1265. doi:10.1007/s00429-018-01826-0

Root, D. H., Ma, S., Barker, D. J., Megehee, L., Striano, B. M., Ralston, C. M., . . West, M. O. (2013). Differential roles of ventral pallidum subregions during cocaine self-administration behaviors. J Comp Neurol, 521(3), 558-588. doi:10.1002/cne.23191

Saad, W. A., Luiz, A. C., De Arruda Camargo, L. A., Renzi, A., \& Manani, J. V. (1996). The lateral preoptic area plays a dual role in the regulation of thirst in the rat. Brain Res Bull, 39(3), 171-176. doi:10.1016/0361-9230(95)02089-6

Shreve, P., Uretsky, N. J. P. B., \& Behavior. (1989). AMPA, kainic acid, and N-methyl-Daspartic acid stimulate locomotor activity after injection into the substantia innominata/lateral preoptic area. 34(1), 101-106.

Shreve, P. E., \& Uretsky, N. J. (1991). GABA and glutamate interact in the substantia innominata/lateral preoptic area to modulate locomotor activity. Pharmacol Biochem Behav, 38(2), 385-388. doi:10.1016/0091-3057(91)90296-e

Subramanian, S., Reichard, R. A., Stevenson, H. S., Schwartz, Z. M., Parsley, K. P., \& Zahm, D. S. (2018). Lateral preoptic and ventral pallidal roles in locomotion and other movements. Brain Struct Funct, 223(6), 2907-2924. doi:10.1007/s00429-018-1669-2

Swerdlow, N. R., Vaccarino, F. J., Amalric, M., \& Koob, G. F. (1986). The neural substrates for the motor-activating properties of psychostimulants: a review of recent findings. Pharmacol Biochem Behav, 25(1), 233-248. doi:10.1016/0091-3057(86)90261-3

Weissbourd, B., Ren, J., DeLoach, K. E., Guenthner, C. J., Miyamichi, K., \& Luo, L. (2014). Presynaptic partners of dorsal raphe serotonergic and GABAergic neurons. Neuron, 83(3), 645-662. doi:10.1016/j.neuron.2014.06.024

Yetnikoff, L., Cheng, A. Y., Lavezzi, H. N., Parsley, K. P., \& Zahm, D. S. (2015). Sources of input to the rostromedial tegmental nucleus, ventral tegmental area, and lateral habenula compared: A study in rat. J Comp Neurol, 523(16), 2426-2456. doi:10.1002/cne.23797

Zahm, D. S., \& Root, D. H. (2017). Review of the cytology and connections of the lateral habenula, an avatar of adaptive behaving. Pharmacol Biochem Behav, 162, 3-21. doi:10.1016/j.pbb.2017.06.004

Zahm, D. S., Schwartz, Z. M., Lavezzi, H. N., Yetnikoff, L., Parsley, K. P. J. B. S., \& Function. (2014). Comparison of the locomotor-activating effects of bicuculline infusions into the preoptic area and ventral pallidum. 219(2), 511-526. 
bioRxiv preprint doi: https://doi.org/10.1101/2021.05.29.446307; this version posted May 30, 2021. The copyright holder for this preprint (which was not certified by peer review) is the author/funder, who has granted bioRxiv a license to display the preprint in perpetuity. It is made available under aCC-BY-NC-ND 4.0 International license.

15

Figure 1.

A

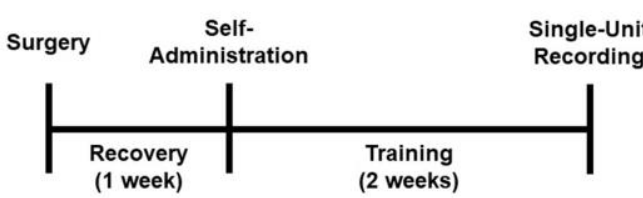

B
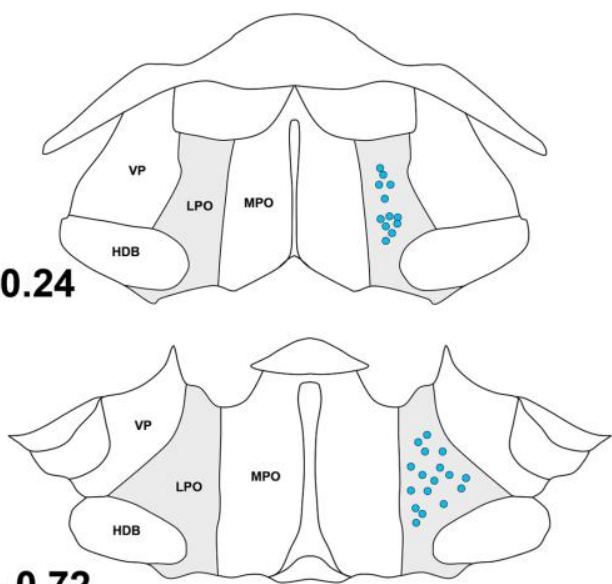

$-0.72$

C
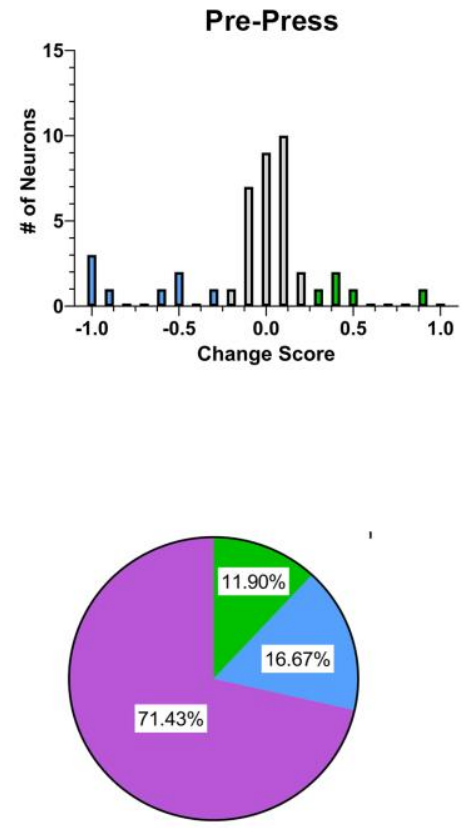

Pre-Press $(\mathrm{N}=42)$
D
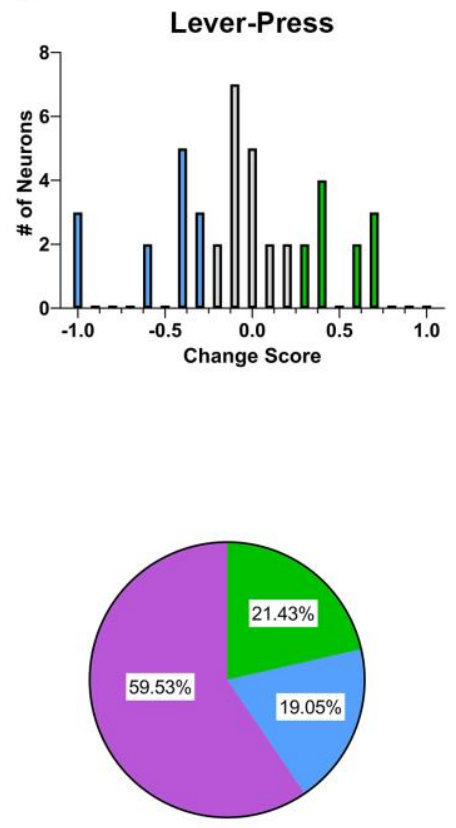

Lever-Press $(n=42)$
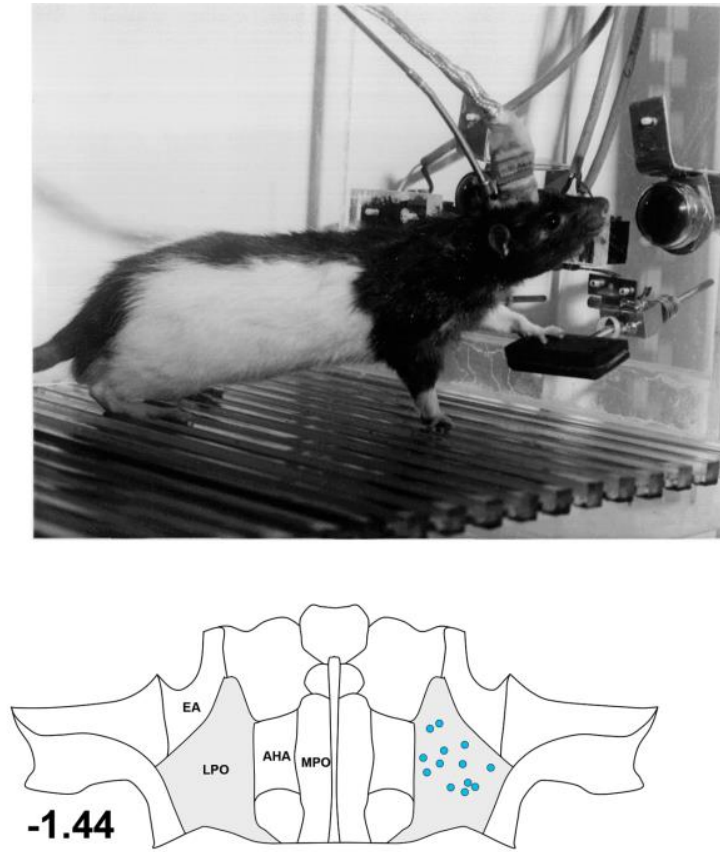

E

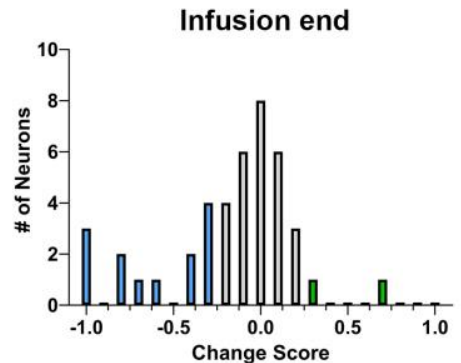

Increase

$\square$ Decrease

$\square$ No Change

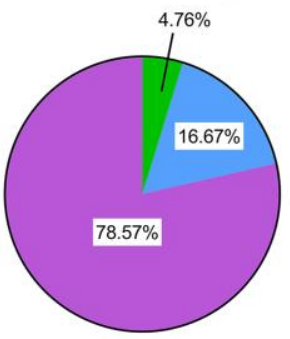

Infusion end ( $n=42)$ 


\section{Figure 1. Lateral preoptic area neurons respond to multiple-aspects of cocaine self-}

administration behavior. A) Rats were implanted with an intravenous catheter and then trained to self-administer cocaine for two-weeks prior to electrophysiological recordings of lateral preoptic neurons. B) A total of 42 neurons were localized within the borders of the lateral preoptic area. Each blue dot represents the localized tip of a single microwire. C) LPO neurons were active during the pre-press window $(\mathrm{t}(41)=5.74, \mathrm{p}<0.001)$, with approximately equal numbers of neurons exhibiting pre-press increases (11.90\%) or decreases (16.67\%). D) LPO neurons were most responsive at the time of lever-responding $(\mathrm{t}(41)=7.52, \mathrm{p}<0.001) . \sim 40 \%$ of recorded neurons exhibited responses specific to the lever response. The majority of these were activated at the time of the lever press $(21.43 \%)$, although other neurons were inhibited at the time of the lever response (19.05\%). E) The smallest subset of neurons were found to be responsive following the end of the infusion $(\mathrm{t}(41)=6.07, \mathrm{p}<0.001)$. These responses were heavily biased towards inhibition (16.67\%), with only a small subset of neurons showing postinfusion activation $(4.76 \%)$. 
Figure 2.

A

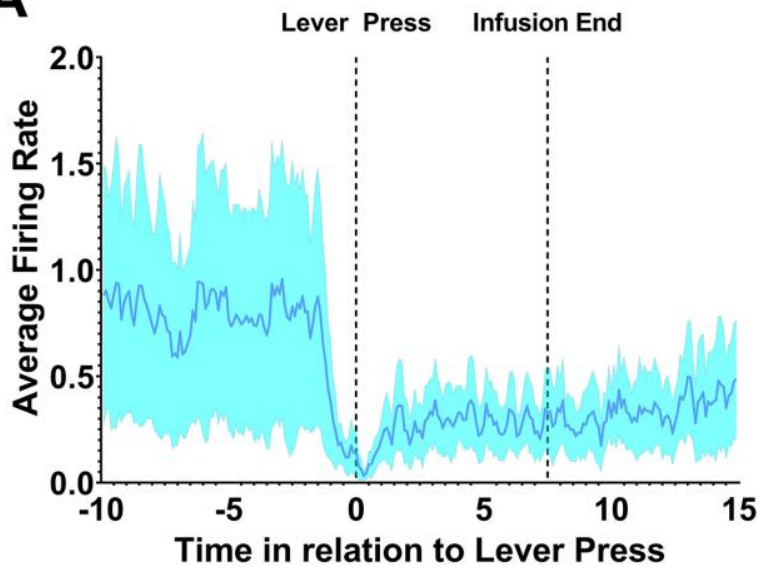

C

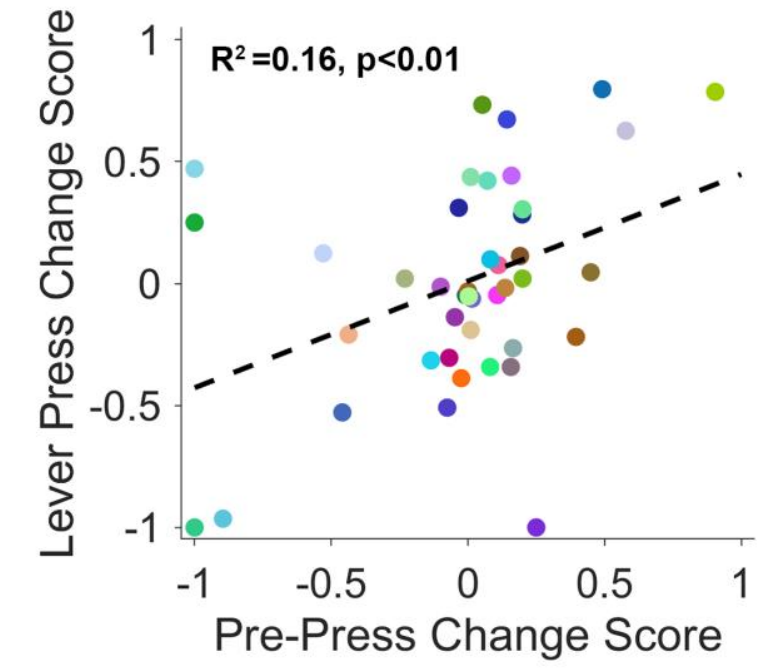

E

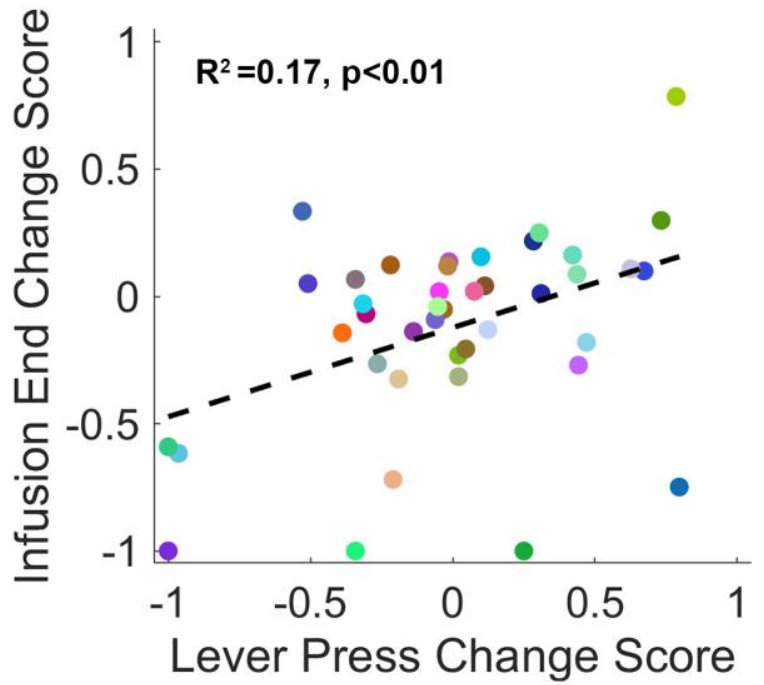

B

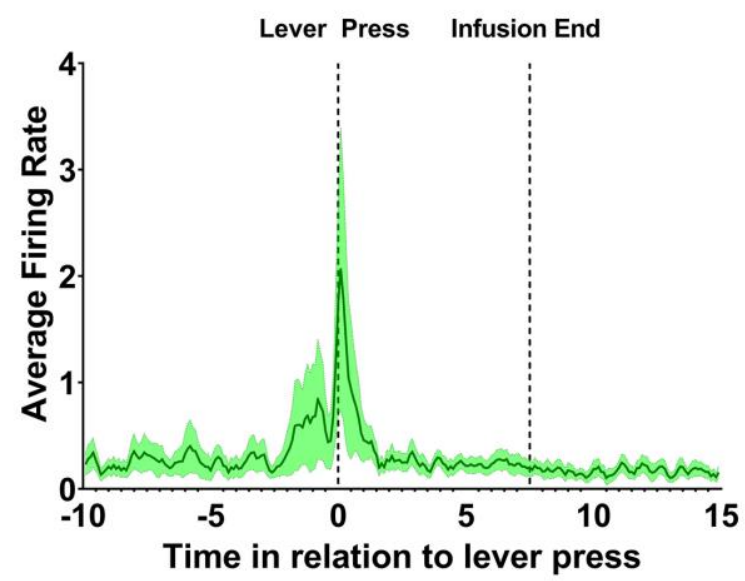

D

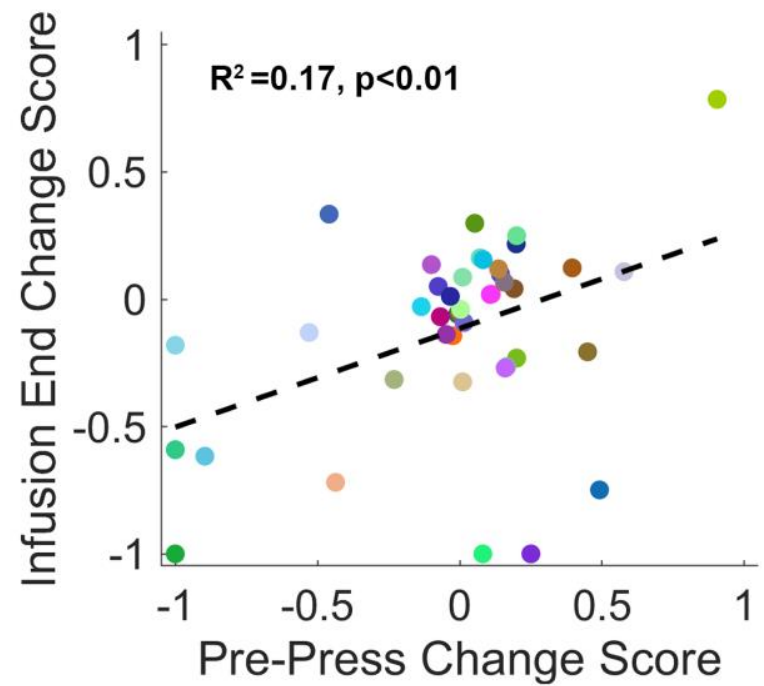

$\mathbf{F}$

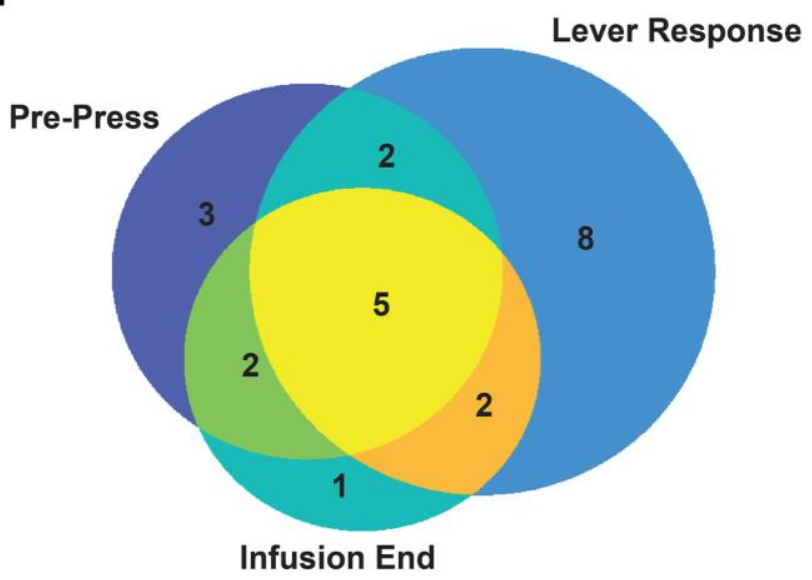




\section{Figure 2. The responses of lateral preoptic area neurons are tied to operant responding. A)}

Averaging neurons exhibiting an inhibitory response at the time of the lever response revealed peri-response inhibition, followed by a long-lasting inhibitory response. B) Averaging neurons exhibiting an excitatory response at the time of the lever response revealed peri-response excitation that was short lived, starting during the pre-press window and extending until just after the lever response. C-E) Consistent with the observation of common response patterns across multiple events, we observed that responding during the pre-press, lever press, and infusion end windows was highly correlated. Each data point is color-coded for specific neuron of interest to allow for cross-comparison. F) In cross-examining responsive, it became clear that responses were most heavily tied to the operant response, with some responses beginning just prior to the operant response and a small number extending across the length of the infusion period. 